\title{
NARRACIÓN DE ESTUDIANTES DE PEDAGOGÍA ANTE EL PROCESO DE INGRESO A LA UNIVERSIDAD ${ }^{I}$
}

\author{
Antonio Freire Hermosilla , Nelson Rodríguez Arratia ${ }^{* * *}$, Pablo \\ Solórzano Marchant ${ }^{\text {t*t }}$
}

\begin{abstract}
RESUMEN:
Este artículo intenta dar cuenta de las motivaciones y dificultades que tuvieron estudiantes de la UCSH en su proceso de ingreso a esta casa de estudios. Para algunos el ingreso a la educación superior significaba la realización de un proyecto de vida personal. Sin embargo, los bajos resultados obtenidos en la PSU se convirtieron en una dificultad para realizar dicho anhelo. La alternativa de estudiar pedagogía se vislumbró como la única posibilidad para obtener un título profesional. La decisión de ingresar en la UCSH estuvo fuertemente marcada por algunas características identitarias y criterios fundacionales de esta universidad. En particular, la acogida y la cordialidad que experimentaron en el proceso de admisión se constituyeron en un apoyo importante para tomar su decisión.
\end{abstract}

Palabras clave: Narrativas - proyecto de vida - proceso de ingreso - características identitarias criterios fundacionales.

\section{ACCOUNT OF A PEDAGOGY STUDENT ABOUT THE PROCESS OF ENTERING THE UNIVERSITY}

\begin{abstract}
:
This article intends to present the motivations and difficulties that some UCSH students had in their process of entering the University. For some of them, to become a student of the higher education meant the achievement of a personal life project. However, the low results they obtained in the PSU (University Selection Test) became an obstacle to reach this goal. The alternative of studying pedagogy is distinguished as the only option to have a degree. The decision of entering the UCSH was strongly determined by some identity characteristics and foundational criteria of this University. Particularly, the reception and cordiality they experience in the admission process became a significant support to have taken the decision.
\end{abstract}

Keywords: Narratives - life project - entering process - identity characteristics - foundational criteria.

I Proyecto de investigación Semilla. DIPOS/ DHEM/ P. EN FILOSOFÍA. UCSH. 2009.

* Freire Antonio, Licenciado en Filosofía. I983 U.P.S. Roma/ Magíster en Filosofía. 2004. Universidad de Chile. Adscrito a UCSH. antofreire@hotmail.com

* Rodríguez Nelson, Profesor de Filosofía: Licenciado en Educación, 1997, UCSH/ Magíster en Letras, Mención Literatura Universal. 200I. PUC/ Doctorado en Filosofía (E), Mención Estética y Teoría del Arte. 2005. U. de Chile Adscrito a UCSH. arronelaire@gmail.com

* Solórzano Pablo, Profesor de Filosofía: Licenciado en Educación, 200I, UCSH/ Magíster en Educación. Mención Gestión y Liderazgo Pedagógico. 2010. UCSH/ Doctorado en Ciencias de la Educación (D). 2009. PUC. Adscrito a UCSH. psolorzanom@ucsh.cl 


\section{Introducción}

La educación se ha instalado como un tema recurrente en la discusión social de este tiempo. En ella toman parte diversos espectadores del quehacer nacional. Sin embargo, llama la atención que la opinión de los principales protagonistas del fenómeno educativo no tenga un reconocimiento y una influencia en las determinaciones políticas que este proceso conlleva. Se discute con vehemencia sobre la profesión docente sin que los profesionales de la educación y los principales destinatarios de ella intervengan en el debate.

Teniendo en cuenta esta preocupación, nos pareció relevante recoger los relatos que ofrecen los propios estudiantes acerca del quehacer docente y la imagen del profesor y su profesionalidad. Para llevar a cabo esta tarea, hemos formulado un proyecto que se enmarca en la comprensión de los discursos de estudiantes de los programas de pedagogía de primer año del departamento de humanidades de la UCSH. Nos interesaba descubrir e interpretar el sentido de la profesión docente a partir de los diversos significados atribuidos por estos estudiantes recién egresados de la enseñanza media.

El proyecto llevaba por título "Relatos y narrativas de estudiantes de pedagogía en torno al sentido de la profesión docente. Un aporte a la relación educativa para los docentes universitarios", y tenía por finalidad rescatar la construcción de sentido del ejercicio pedagógico. Para esto, formulamos la propuesta de comprensión desde dos perspectivas diferentes, pero complementarias: el análisis actancial de Greimas y la fenomenología hermenéutica de Paul Ricoeur.

El presente artículo se centra en el Proceso de acceso a la formación superior por parte de estudiantes de pedagogía del Departamento de Humanidades y Enseñanza Media de la UCSH. En principio nos interesa descubrir las motivaciones que llevan a los estudiantes a optar por la Universidad Católica Silva Henríquez. 
Un primer motivo que esgrimen para estudiar pedagogía está vinculado con ciertas cualidades $y$ habilidades personales ${ }^{2}$ que desean colocar al servicio de los demás. Si bien, la elección de la carrera no está clara en muchos de ellos, para algunos el ingreso a la universidad les ayuda a descubrir, clarificar y potenciar su vocación, que al inicio se encuentra difusa. De esta manera, el ingreso a la universidad se convierte en un medio para realizar su proyecto personal.

En un primer momento, su deseo era ingresar en universidades públicas. Sin embargo, la baja ponderación de sus puntajes en la PSU se convirtió en una dificultad para realizar dicho anhelo. De ahí que la opción por ingresar a una carrera profesional quedó restringida a pocas alternativas.

En general, para nuestros estudiantes, sus insumos para ingresar a una carrera profesional no eran los óptimos. Al no tener el puntaje exigido para la carrera que querían estudiar, tuvieron que resignarse a buscar en una universidad privada un cupo que les permitiera ingresar a la educación superior, sin ver afectada su capacidad de pago.

Al optar a una carrera de pedagogía lo hicieron ya sea por decisión propia o por descarte. En la elección de esta universidad influyeron varios factores; uno fue la acogida que se experimentó en el proceso de inscripción; otro fue el hecho de estar acreditada, ya que de esta forma los estudiantes podían acceder a un crédito con aval del Estado. Otro factor fue el reconocimiento de la marcada dimensión pedagógica de la UCSH. Este sello identitario de la universidad constituye una fortaleza que los mismos estudiantes iniciales han percibido en el ámbito escolar a nivel local.

Si bien la decisión de estudiar pedagogía en esta universidad puede ser por azar, descarte o convicción, no se puede desestimar ninguna de ellas, ya que se trata de un ejercicio que evalúa las condiciones de posibilidad dadas por el puntaje en la PSU, el crédito con aval del Estado y las características identitarias de la universidad.

2 “... siempre tuve la inquietud de enseñar, y me gustó, siempre tuve la, por llamarlo así, tuve la habilidad de explicar las cosas complicadas fácilmente”. P. I4. Focus - Group 
La presente publicación incluye el marco referencial, el diseño metodológico, un ejercicio de análisis actancial, otro de análisis hermenéutico y las conclusiones.

\section{Marco referencial}

La Congregación Salesiana y la Universidad Católica Silva Henríquez, a través de su proyecto de formación, han optado por un modelo de inclusión de sus alumnos, por sobre la exclusión o selección de los mismos. Lo anterior busca asegurar plenamente un proyecto de formación que desde la experiencia carismática y de gestión, promueva la formación de profesionales de calidad, a partir de sus aprendizajes y expectativas de resultados. En este sentido, la inclusión de los estudiantes se realizaría bajo sus supuestos fundacionales, lo que implicaría su acogida y no la selección de procesos de postulación estandarizados.

Por otra parte, hoy se plantea que a pesar de la fuerte inversión financiera que han realizado muchos países en educación para mejorar la equidad social por intermedio de la calidad educativa, no han significado una mejora sustancial (McKinsey, 2007). Por otro lado, se ha privilegiado la intervención y la administración de los recursos, que logre posibilitar una estructura sistémica desde un liderazgo adaptativo (Hopkins, 2008). El informe de Hopkins concluye que el proceso de acompañamiento sistémico para la formación de profesores es la piedra angular para su mejoramiento.

Para la gran mayoría de estos estudiantes de esta investigación, la pedagogía no es su primera opción vocacional. Sus modestos resultados en la P.S.U. no constituyen una ventaja respecto a su opción preferencial. También, dado su origen socio-económico, existe una fuerte desesperanza adquirida que los lleva a optar por carreras pedagógicas con un cierto desinterés. Por otra parte, se debe considerar que en el medio social no se tiene una ponderación positiva de la profesión docente, que lleva a que ésta no cuenta con el reconocimiento que sí tienen otras profesiones mejor catalogadas 
Desde la perspectiva de Paul Ricoeur, todo ser humano tiene la posibilidad de expresar en su lenguaje y acciones, su vida. Así, puede atender a las dificultades y resolver desde un modo de ser particular y en lo social, pues decide, elige y siente sus decisiones como modos de vida para adquirir un sentido vital, una orientación en el mundo que es el propio. No existe separación entre él y su interpretación (sujeto-objeto). Por esta razón, se propone investigar, en primer lugar bajo el paradigma Analítico Estructural (Greimas), para luego desarrollar un segundo momento: el paradigma hermenéutico interpretativo (P. Ricoeur).

En las narrativas (de los estudiantes) se reconoce el carácter performativo de los discursos. Desde ellas, el aspecto biográfico y el contexto socio-cultural de los estudiantes permiten a los docentes universitarios abrir un espacio comprensivo sobre las motivaciones, competencias y sentidos vocacionales y profesionales, hasta ahora no formalizados en la relación educativa o en los perfiles ya existentes en cada uno de los programas de estudio. Narrar la vida es un modo de narrarse, o sea, decirse o hablar de sí mismo. Por lo que cada narrativa abre a los docentes universitarios y a los mismos estudiantes, un horizonte de comprensión en la forma de mirarse más allá de los contextos y formalidades establecidas (Gadamer, 1998).

\section{Diseño metodológico}

Los métodos que se utilizaron se inscriben en el paradigma cualitativo; éste busca profundizar en la comprensión de un fenómeno particular, estudio de caso, que aparece como problemático. El tipo de investigación es exploratorio, que por sus características, establece el tono de investigaciones posteriores más rigurosas (Dankhe, I986, p.p 4I2); en este sentido la exploración busca abrir nuevos frentes de significaciones y problemáticas atingentes, que permitan levantar una línea de investigación desde la 'Fenomenología Hermenéutica'.

La propuesta investigativa se sustenta desde dos planteamientos teóricos que intentan descubrir e interpretar el sentido de los 
discursos. El primero, la Fenomenología Hermenéutica, se configura desde los principios teóricos del filósofo francés Paul Ricoeur, esto es, la articulación de sentido narrativo en 'el mundo de la vida' y la sistematización teórica sobre 'representaciones sociales'; se inscribe en la tradición que desde la vertiente sociológica busca regularidades en los discursos de los sujetos para descubrir sentidos en la interacción social. El segundo corresponde el Análisis Estructural de Greimas (Martinic, 2005).

La Fenomenología Hermenéutica procura comprender discursos de sentido que para la lógica de las ciencias son considerados equívocos. Pero desde esta perspectiva no es un error del pensamiento, ya que contiene una 'doble lógica' que invita a su comprensión segunda para develar su significado desde el 'símbolo', 'la metáfora' y 'el texto' (Ricoeur,1987). Los supuestos parten de la base de que no existe una separación radical entre 'texto, contexto y sujeto' y que el relato narrativo se identifica semánticamente con el 'mundo de la vida'. La configuración del relato está cargada de significado y sentido en la traducción de las historias personales, y en la distancia de tiempo y en la conciencia se realiza una traducción de ficción e intriga (Ricoeur, 1984).

Desde un punto de vista epistemológico, la 'representación social' se puede entender como un fenómeno que condensa un conjunto de significados: 'categorías que sirven para clasificar las circunstancias, los fenómenos y a los individuos con quienes tenemos algo que ver; categorías que permiten establecer bechos sobre ellos' (Jodelet, I986, p.p 56).

Para el objeto de estudio de esta investigación, se hace necesario utilizar estas dos líneas teóricas que permiten abrir la comprensión de las narrativas de los estudiantes desde el texto. Si bien la fundamentación se origina desde los postulados de la Fenomenología Hermenéutica, el inicio operativo de la traducción de sentidos en los discursos se realiza desde las 'representaciones sociales' para que, en otro momento, se desarrolle una reflexión segunda de tipo hermenéutico. 


\section{Análisis actancial}

I. Categorías construidas y modelo simbólico con respecto al Proceso de ingreso a la Universidad.

I. Categorías y ejes construidos.

TI. Condición ideal para optar a una carrera profesional.

cA: Opción para estudiar en una universidad pública: bien en PSU ${ }^{3}$ / estudiar por convicción / por ser universidad pública / suficiente preparación personal para la PSU.

cB: Opción para estudiar en una universidad privada: mal en PSU / estudiar por hacer algo / por ser una alternativa / escasa preparación personal para la PSU.

T2. Condición real de estudiantes de la UCSH para decidirse por una carrera profesional.

cA: Elección por Universidad privada: por descarte ${ }^{4}$ / porque no me fue bien en PSU / por ser alternativa viable / estudiar lo que sea.

cB: Elección por Universidad pública: por mérito / porque me fue bien en PSU / por ser lo que quería / estudiar lo que elijo.

3 Sí, emmm... como no me fue muy bien en la PSU obviamente tuve que optar a una universidad privada, lamentablemente no me preparé, como no tomé conciencia en ese momento de querer estudiar 'para prepararme para estudiar para la prueba, entonces fue mi primera opción llegar acá, ya que era una universidad privada, no hay mucho problema como para entrar, con un puntaje relativamente bajo... emmm, por eso opte acá, porque he escuchado harta, o sea, muy buenas críticas, sobre todo de pedagogía, entonces eso es como lo que más me interesó. Ped. Artes. E.2 P.25.

4 "Eee, los pasos que se podrían decir no sé, igual yo postulé a esta carrera porque no logré quedar en el pedagógico, pero como te decía anteriormente, siempre he tenido esa convicción de que aunque mi madre me decía ":Estay seguro que querí estudiar filosofía, tay seguro?” y yo sí desde muy chico, o sea no desde muy chico, desde segundo medio, hace cuatro años no más, igual siempre fue, me costó igual llegar a decidirme bien por filosofía, pero no por un tema de no convicción propia, sino porque no podía entrar donde yo quería”. Ped. Fil.E.I. P.52. 
Narración de estudiantes de pedagogía ante el proceso de ingreso a la universidad - Antonio Freire, Nelson Rodríguez y Pablo Solórzano

T3. Percepción social de la dificultad de cursar la carrera de pedagogía en la UCSH.

cA: Condición real de estudiar pedagogía: pega jodida ${ }^{5}$ / le da pena frente a las malas evaluaciones / difícil de estudiar / se enseña frente a gran cantidad de estudiantes.

cB: Prejuicio social de la pedagogía: pega de fácil ejecución / juzga al profesor frente a las evaluaciones / fácil estudiar /se puede enseñar no dependiendo de la cantidad de estudiantes.

\title{
2. Cruzadas y campos de sentido con respecto al proceso de ingreso a la universidad
}

I. Condiciones ideales para optar a una carrera profesional

\author{
TIPOS DE \\ UNIVERSIDADES \\ Al \\ Universidad Pública
}

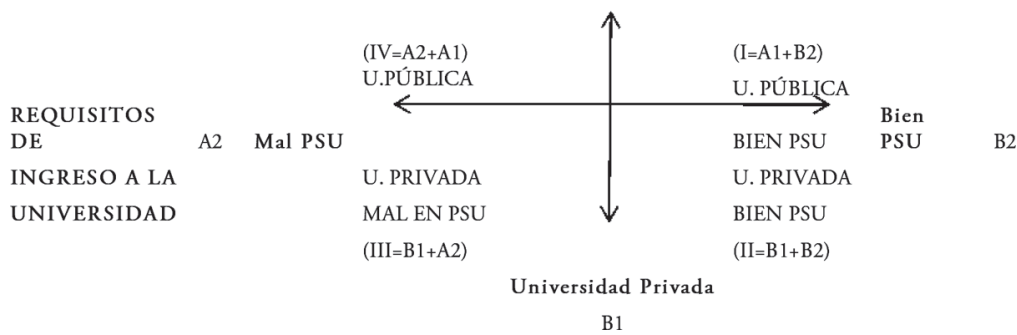

(Figura I)

El cuadro muestra cómo los estudiantes identifican las condiciones ideales para optar a una carrera profesional, dependiendo del tipo de universidad y su puntaje en la Prueba de Selección Universitaria. Los cuatro campos de sentido que se generan son:

5 "bastante, es reafirmar aparte de lo que me gusta, lo que me gusta hacer, lo que me gusta enseñar, me ha enseñado muchos valores también, a no ser egoísta verdad, a ser mucho más paciente, a ser tolerante, perseverante, eso es lo más importante yo creo, eso es lo que ahora llevo muy poquitito en realidad en la carrera, pero ya me estoy adecuando y más que nada es eso, que me está enseñando a la vez más a valorar la pedagogía que es bastante difícil o como dicen pega jodida jajajaj (risas), pero me ha enseñado muchas cosas y sobre todo esto la tolerancia, la perseverancia”. Ped. Artes. E.2. P.33. 
$\mathrm{I}(\mathrm{AI}+\mathrm{B} 2)=$ se expresa que la condición ideal para optar a una carrera profesional es cuando un estudiante accede a una carrera profesional en una 'universidad pública', cuando le va 'bien en los resultados de la PSU'.

II $(B I+B 2)=$ se expresa que también es posible acceder a una carrera profesional, aunque no de manera ideal, a una 'universidad privada', a pesar de que sus resultados sean 'buenos en PSU'.

$\mathrm{III}(\mathrm{BI}+\mathrm{A} 2)=$ se expresa que también es posible acceder a una carrera profesional, aunque de manera negativa, a una 'universidad privada' condicionado por los 'malos resultados de la PSU'.

IV $(A 2+A I)=$ se expresa que también es posible acceder a una carrera profesional en una 'universidad pública' a pesar que los 'resultados de la PSU fuesen no muy buenos'.

En los discursos de los estudiantes se aprecia lo que consideran como las condiciones ideales para acceder a estudiar una carrera profesional. En este sentido se valora positivamente $(++)$ el ingreso a estudiar en una 'universidad pública', lo que significa que huyan rendido una buena PSU. Para acceder se valora 'la preparación en la PSU', junto también con la convicción por la carrera.

Por otra parte, al contrario, se considera que las condiciones negativas (--) para acceder a estudiar una carrera profesional son que se estudie en una 'universidad privada', y que el puntaje de la PSU haya sido muy insuficiente. En este sentido potencia esta decisión 'la falta de preparación en PSU', junto con elegir la carrera 'por hacer algo'.

Un posible campo de sentido que se abre (+-) es que los estudiantes puedan acceder al estudio de una carrera profesional en una universidad pública a pesar de que no les haya ido bien en la PSU. También, otro campo de sentido (-+), sería que a pesar que en el puntaje de PSU les haya ido bien, elijan estudiar en una universidad privada. 


\section{Condición real de estudiantes de la UCSH para decidir por una carrera profesional.}

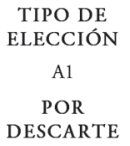

El cuadro muestra cómo los estudiantes se ven enfrentados al modo de decisión de una carrera profesional con respecto a las condiciones reales para tomarla, en este caso por una convicción propia por la carrera o porque es una de otras alternativas. Los cuatro campos de sentido que se generan son:

$\mathrm{I}(\mathrm{AI}+\mathrm{B} 2)=$ se expresa que la condición real para decidir por una carrera profesional, se produce por medio de un 'descarte', lo cual a diferencia de la elección por otras carreras, puede ser una 'alternativa viable'.

II $(B I+B 2)=$ se expresa que la condición real para decidir por una carrera profesional, se produce por medio del 'mérito', lo cual a diferencia de la elección por otras carreras, puede ser una 'alternativa viable'.

III $(B I+A 2)=$ se expresa que la condición real para decidir por una carrera profesional, se produce por medio del 'mérito', lo cual es una 'alternativa propia'.

IV $(\mathrm{A} 2+\mathrm{AI})=$ se expresa que la condición real para decidir por una carrera profesional, se produce por medio de un proceso de 'descarte', lo cual puede ser también una 'alternativa propia'. 
En los discursos de los estudiantes se aprecia lo que consideran los estudiantes como las formas reales que están implicadas a la hora de decidir estudiar una carrera profesional. En este sentido se valora positivamente $(++)$ como una condición real que los estudiantes deciden ingresar a una carrera profesional por descarte y porque puede ser una alternativa viable dentro de las pocas alternativas.

Por otra parte, al contrario, se considera que las condiciones negativas (--) para decidir estudiar una carrera profesional es que sea por mérito y por alternativa propia, o por su propia voluntad.

Un posible campo de sentido que se abre (+-) es que la decisión se realiza producto de una alternativa propia, a pesar de que se haya elegido por descarte. También, otro campo de sentido (-+) que se abre, sería aquel, que teniendo mérito para ingresar a una carrera de pedagogía, encuentra que estudiar pedagogía es una alternativa viable.

\section{Realidad de estudiar pedagogía en comparación} con la apreciación social.

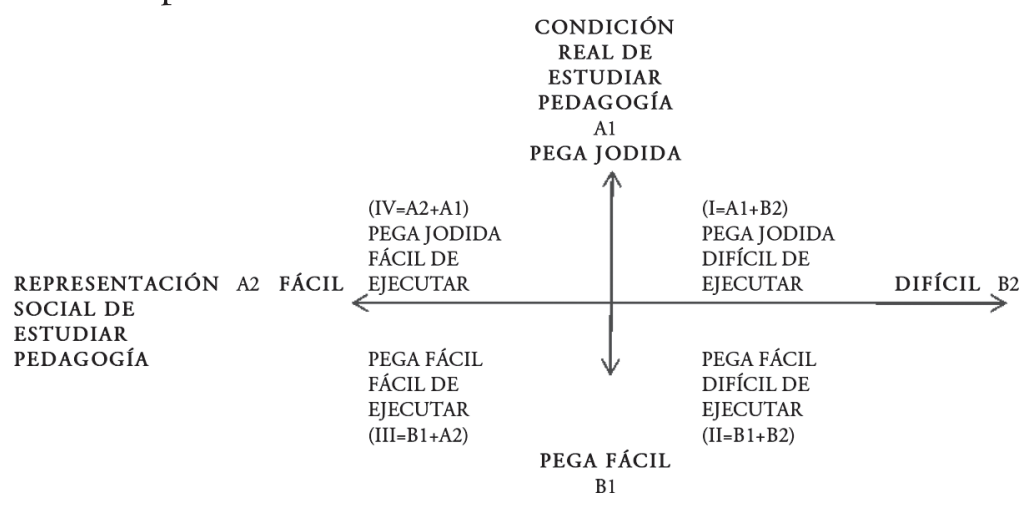

(Figura 3)

El cuadro muestra la apreciación que tienen los estudiantes sobre la realidad de estudiar pedagogía, con respecto a la representación del esfuerzo que amerita el trabajo en este campo. En este sentido los cuatro campos que se generan son: 
Narración de estudiantes de pedagogía ante el proceso de ingreso a la universidad - Antonio Freire,

$\mathrm{I}(\mathrm{AI}+\mathrm{B} 2)=$ se expresa que la condición real de estudiar pedagogía es que se puede denominar como una pega jodida y difícil de ejecutar.

II $(B I+B 2)=$ se expresa que también es posible que sea considerada una pega fácil pero de difícil ejecución.

$\mathrm{III}(\mathrm{BI}+\mathrm{A} 2)=$ se expresa que también es posible que se considere como una pega fácil y fácil de ejecutar.

IV $(\mathrm{A} 2+\mathrm{AI})=$ se expresa que también se puede considerar como una pega jodida, pero fácil de ejecutar.

En los discursos de los estudiantes se aprecia lo que consideran como las condiciones reales de estudiar pedagogía, en este sentido el campo positivo $(++)$ aparece que es una pega jodida y difícil de ejecutar. Esta cuestión de conocimiento de parte de los estudiantes permite plantear que frente a las evaluaciones la figura del profesor le da pena; además trabajan con 45 alumnos en el aula.

Por otra parte, al contrario, se considera que las condiciones negativas (--) desde una mirada social que tiene el desconocimiento de la realidad de los profesores, es que se puede considerar como una pega fácil, de fácil ejecución.

Un posible campo de sentido que se abre (+-) es que la condición real del trabajo sobre la pedagogía sea entendida como una 'pegajodida' y a la vez, un trabajo de fácil ejecución. También, otro campo de sentido (-+), sería que se considere a la educación como una 'pega fácil' pero difícil de ejecutar.

\section{Principios simbólicos y acciones sociales}

\section{Descripción del modelo de acción}

Búsqueda del sujeto del bien deseado. Relación Sujeto - Objeto

En la representación de los actores, en este caso de estudiantes de pedagogía con respecto a elección real de estudiar pedagogía, en la figura 4 su alternativa existencial se define según la dicotomía: 
Estudiante que elige lo que quiere $(+)$ o Estudiante que elige lo que sea (-). La disyunción planteada constituye el sujeto positivo y negativo respectivamente.

MODELO DE ACCIÓN SIMBÓLICA

Destinador +

Se aprecia al profesor por su desempeńo

Es valorada la profesión docente

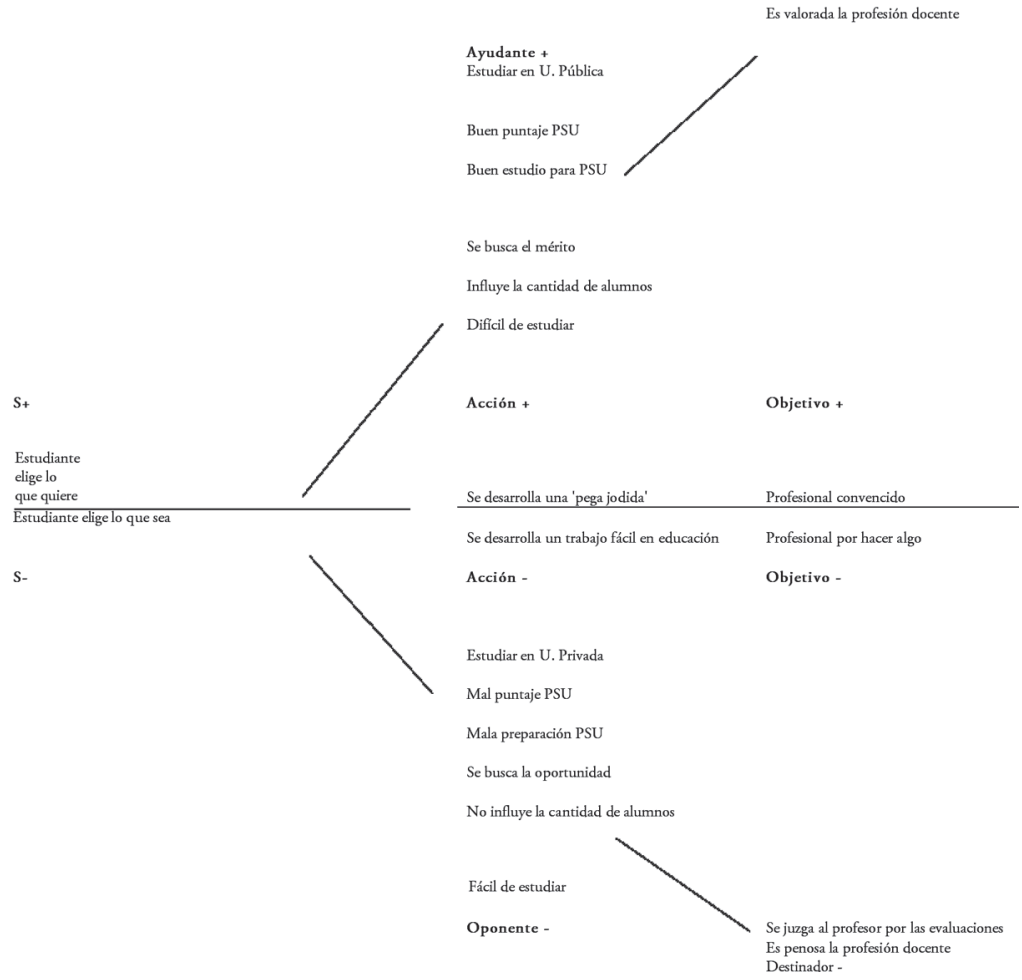

(Figura 4)

El origen de esta alternativa existencial se encuentra en la idea de que los estudiantes de pedagogía tienen posibilidades de elegir la carrera, lo que conllevaría una serie de acciones y ayudantes, esto permitiría que el estudiante sea un profesional convencido.

\section{Las acciones para llegar al objeto positivo}

En el mismo eje anterior, se encuentra la alternativa de acción que desde el punto de vista del actor, permite alcanzar el bien o el objetivo 
deseado. En el lado positivo se advierte la idea que estudiar una carrera en pedagogía es desarrollar una pega jodida, cuando ya se ha tenido alguna experiencia, no se considere como una cuestión fácil. Al contrario, se advierte en la percepción social que se entienda el estudiar pedagogía como una cuestión fácil, lo que conlleva a que el trabajo en sí mismo sea fácil.

\section{Ayudantes y oponentes}

En el proceso de ir constituyendo la experiencia de cursar una carrera de pedagogía, se entiende que el estudiante que elige la carrera termina siendo un profesional convencido (objetivo deseado-O+) de la profesión que estudió. Para que esto suceda, las acciones positivas son que asuma la realidad de estudiar pedagogía, lo cual es difícil, pero tiene una serie de ayudantes para que esto suceda. Estos ayudantes son que pueda estudiar en una 'universidad pública', que tenga un 'buen puntaje en PSU' y para que esto suceda, debe haberse preparado, junto con 'buscar el mérito'. Por el contrario, las acciones negativas que irían en contra de este objetivo, generando un profesional por hacer algo, (O-), serían que estudie en una 'universidad privada', que no le hubiere ido bien en la PSU, que no se haya preparado para estos fines, y que no busque el mérito en la profesión.

\section{La comunicación del destinador hacia el destinatario}

En los textos se encuentran elementos existentes que comunican y otorgan al sujeto un poder para desencadenar o avalar una acción. El destinador en este estudio incluye la potencialización en la representación social del elegir estudiar una carrera de pedagogía, lo que sin la experiencia de cursarla, no permitiría 'apreciar al profesor por su desempeño' como también, 'valorar la profesión docente'. Por el contrario, cuando no se tiene la experiencia y el estudiante la 'elige por hacer algo', da paso a que la pedagogía se siga 'juzgando por las evaluaciones a profesores', junto con entenderla como una 'profesión penosa'. 


\section{Análisis hermenéutico}

\section{El proceso de ingreso a la universidad}

La educación media en Chile está fuertemente enfocada a la educación superior, ya sea en institutos profesionales, centros de formación técnica y universidades estatales o privadas. En la investigación interesa descubrir las motivaciones que llevan a los estudiantes a optar por la Universidad Católica Silva Henríquez.

De acuerdo a lo que declararon los estudiantes en el Focus-Group realizado, un primer motivo que esgrimen para estudiar pedagogía está vinculado a contar con ciertas cualidades y habilidades personales ${ }^{6}$ que pueden colocar al servicio de los demás. Este primer motivo tiene su base en la experiencia de vivencias que han tenido en la Enseñanza Media. Si bien, la elección de la carrera no está clara en muchos de ellos, para algunos el ingreso a la universidad les ayuda a descubrir, clarificar y potenciar su vocación, que en un principio se encuentra difusa. En este sentido, el ingreso a la universidad se convierte en un medio para realizar su proyecto personal.

En principio, el ideal de gran parte de los estudiantes egresados del sistema municipal y particular subvencionado, desde donde proviene la mayoría de los estudiantes recién ingresados a la UCSH, es la opción por universidades públicas. Sin embargo, la baja ponderación de sus puntajes en la PSU se convierte en una dificultad para realizar dicho anhelo. Esto los lleva a optar por una universidad privada ya que en ella existe una posibilidad real de que puedan realizar sus proyectos. En este sentido la elección de ingresar a una carrera profesional se mediatiza por sus conciencias y ponen en juego alternativas para optar a una carrera profesional. Se puede plantear, que es posible considerar aquella elección como la alternativa que ellos preferían en primera opción, pero también, considerar la decisión como la alternativa mejor de las pocas posibilidades que tenían para acceder

6 "... siempre tuve la inquietud de enseñar, y me gustó, siempre tuve la, por llamarlo así, tuve la habilidad de explicar las cosas complicadas fácilmente". P. I4. Focus-Group 
Narración de estudiantes de pedagogía ante el proceso de ingreso a la universidad - Antonio Freire, Nelson Rodríguez y Pablo Solórzano

a la educación superior; quizás planteado de otro modo, la única posibilidad para cursar a una carrera profesional.

Frente a esta elección existe un salto frente al optar a una carrera de pedagogía por decisión propia o por descarte; se piensa al observar los datos -los insumos para ingresar a una carrera profesional no son los óptimos- que los estudiantes terminan incorporando narrativas o discursos en su interior que les permiten sostener la elección como oportunidad, para en el tiempo, ir comprometiendo una actitud profesional en un futuro que se anhela con respecto al rol por asumir cuando se adquiera la profesión. Por lo mismo, si bien las condiciones previas para ingresar a la educación superior no son las mejores, la experiencia de cursar la carrera, se va traduciendo en complejidades interpretativas que permiten ir desarrollando una traducción del entorno, el contenido y la vivencia en pro de una profesión con sentido. Hay que hacer notar que la valoración social desprendida del modelo actancial para que el estudiante que eligió la carrera por convicción sea un profesional convencido, tiene como requisito, haberse preparado para la PSU, haberle ido bien, y auto-valorar su desempeño por el mérito, cuestión que en los mismos datos de los estudiantes no se aprecia.

Por otra parte, en el Focus-Group realizado, los estudiantes manifestaban como primera prioridad entrar a una universidad estatal ${ }^{7}$, ya que en esa podían acceder a créditos convenientes para pagar su educación superior $y$, de esta forma, concretizar el deseo de ser profesionales. Al no tener el puntaje exigido para la carrera que querían estudiar, tuvieron que resignarse a buscar en una universidad privada un cupo que les permitiera ingresar a la educación superior, sin ver afectada su capacidad de pago.

Otros de los motivos expresados por los alumnos para preferir las universidades estatales, tiene que ver con el prestigio, el reconocimiento

7 “... a mí me paso algo parecido, yo al igual que ella, no quería estudiar en una privada, en una pública, o sea estatal, por el tema de que joh! La estatal es mejor.” P. 44. Focus-Group 
social y el buen nivel académico de ellas $s^{8}$. De esta forma, piensan que pueden asegurar un campo laboral estable y con mejores expectativas contractuales. Esto no quiere decir que las universidades privadas sean de menor calidad que las estatales, pero influyen fuertemente los prejuicios sociales que se tienen sobre ellas?.

Llama la atención, que uno de los motivos para haber escogido la UCSH, fue la acogida que experimentó parte de los estudiantes. ${ }^{10} \mathrm{La}$ acogida es un elemento importante en las relaciones humanas, ya que por medio de ella se valora a la persona, es decir, se está considerando al estudiante como ser humano y que sí es importante y merece ser atendido como tal.

El estudiante no es un número de matrícula en la universidad, sino una persona que tiene la misma dignidad que todos los seres humanos, independientemente de su condición social, étnica, económica, académica, etc. De la misma manera, la cordialidad manifestada en la convivencia universitaria se constituye en una oportunidad de fortalecer el ingreso y la permanencia de jóvenes y adultos en la UCSH.

Otra variante que influye en la opción por estudiar en la UCSH es la acreditación institucional, puesto que los estudiantes pueden acceder a un crédito con aval del Estado ${ }^{11}$. En muchos casos las familias no tienen o no pueden sostener a un hijo en la universidad por el costo que ella demanda, ya sea por el arancel y por el costo de materiales propios de cada carrera (textos, fotocopias, trabajos, etc.). Una tesis

8 “... a mí me paso algo parecido, yo al igual que a ella, no quería estudiar en una privada, en una pública o sea estatal por el tema de que joh! La estatal es mejor, como uno va al colegio, venís de una privada, y también vi el tema monetario, y comparé y esta es una de las privadas más baratas, y yo estoy con crédito, y entonces pago súper poco, me aceptaban el crédito y te dan beca de alimentación, entonces tenía hartos beneficios...”. P.44. Focus-Group

9 “... en una privada, en la Silva Henríquez, y me dicen jah, no qué guena! Es buena en pedagogía y yo no tengo problemas en decir que estudio en una privada, porque... en aparte uno compara las mallas y son súper parecidas o a veces mejores". P.44. Focus-Group

I0 “... Y fui a la universidad y catetié y pregunté, a mí en la Hurtado a mí siempre, fui como cinco días y me trataron súper mal, mal, onda te vay, qué voy hacer. En cambio cuando llegué aquí y me acogieron súper bien. Y entonces yo dije iya yy! Será mejor y todo el mundo me recomendó esta universidad, porque es buena en pedagogía." P. 46. Focus-Group

II “... eh esto influye el crédito con aval del Estado”. P. 25. Focus - Group “si”. P. 26. FocusGroup 
Narración de estudiantes de pedagogía ante el proceso de ingreso a la universidad - Antonio Freire, Nelson Rodríguez y Pablo Solórzano

realizada en el año 1996 indica entre las tres primeras variables de ingreso a esta universidad -en ese entonces Universidad Católica Blas Cañas- la carrera, el costo y el prestigio (Lira,1996). De acuerdo a lo que declaran los estudiantes ingresados en 2009 , las variables que ellos consideraron para la elección de esta universidad serían el costo, la referencia y el prestigio.

Conviene recordar que uno de los criterios fundacionales de la UCSH ha sido favorecer el acceso a la educación superior a jóvenes provenientes de los sectores más vulnerables de la sociedad. De hecho, esta universidad fue creada para atender a un sector de la sociedad que en su época estaba excluida de la educación superior por su alto costo y difícil acceso.

En el medio nacional hay un reconocimiento por la dimensión pedagógica de la UCSH. Se la valora "porque es buena en pedagogía" ${ }^{2}$. Así opinan las personas que pertenecen al círculo más cercano del estudiante de esta universidad: familiares, vecinos, amigos, etc.

Los estudiantes egresados también estiman y destacan esta fortaleza. Piensan que en su interior, de manera personal, pudieron potenciar sus talentos personales. En el medio laboral, algunos empleadores reiteran y subrayan este aspecto. En efecto, reconocen un sello característico en la actitud de los egresados de esta universidad que actualmente se desempeñan en el campo educativo ${ }^{13}$. Este sello no está oculto, puesto que se manifiesta en las actitudes y comportamiento que tienen los egresados y estudiantes de la UCSH. El sello identitario de la UCSH constituye una fortaleza que los mismos estudiantes iniciales han percibido en el ámbito escolar a nivel local. A su vez, éste tiene una estrecha relación con los talentos que cada estudiante trae consigo al ingresar a esta universidad. En la medida que se reconozcan el

I2 P. 46 Focus-Group

I3 “...jefa de UTP o la encargada de lo, dijo saben qué, han venido chiquillos de la UMCE, han venido chiquillos de otras universidades y no tienen la misma actitud que tú, es que entran a una clase y ven a los cabros desordenados y se van, no tienen como la actitud tuya, incréblemente han venido dos chiquillos de la misma universidad tienen la misma actitud. Eso me da un fiel reflejo de que la universidad está inculcando....”. P. 38. Focus-Group 
sello y los talentos, se fortalece enormemente el desarrollo de las capacidades y habilidades de las personas, es decir, da la impresión que la formación del estudiante en la UCSH es plena, global e integral. Por ello es importante que los estudiantes conozcan este sello identitario. Así, la universidad reconoce los talentos que aportan los propios estudiantes y en esta relación se produce una sinergia que potencia tanto a la universidad como al estudiante.

Si bien la decisión de estudiar pedagogía en esta universidad puede ser por azar, descarte o convicción, no se puede desestimar ninguna de ellas, ya que se trata de un ejercicio que evalúa las condiciones de posibilidad dadas por el puntaje en la PSU, el crédito con aval del Estado y las características identitarias de la universidad. Se tiene en cuenta, por ejemplo, el estar con sus pares en igualdad de condiciones étnicas, comunales, educacionales.

\section{Conclusiones}

El primer atisbo que debemos mencionar en estas conclusiones está referido a la propuesta metodológica de investigación, sustentada en A. Greimas y Paul Ricoeur. El modelo actancial y la fenomenología hermenéutica, nos permitieron acercarnos desde el diálogo a un fenómeno que, en su contenido, nos permite advertir correcciones y modos distintos de proyectar a los alumnos que ingresan a nuestra casa de estudios.

El ingreso de nuestros estudiantes a la UCSH está fuertemente determinado por los resultados en la Prueba de Selección Universitaria (P.S.U.). Claramente ellos son el reflejo de procesos débiles en desarrollo de habilidades, competencias y contenidos adquiridos en su enseñanza anterior, algunos de ellos acentuados, por venir de la educación municipalizada o subvencionada, proveniencia de la gran mayoría los estudiantes de la UCSH. Sin duda, en muchos de nuestros estudiantes, se encuentra la voz de la desazón al momento de ser interrogados por los resultados de P.S.U., pues en su intimidad recorre la posibilidad disuelta de estar en una universidad pública y 
Narración de estudiantes de pedagogía ante el proceso de ingreso a la universidad - Antonio Freire, Nelson Rodríguez y Pablo Solórzano

en otra carrera. Por cierto, se juega aquí gran parte de las expectativas que cada estudiante tiene para sí y en esta casa de estudios.

Si en gran medida, la calidad de un docente en relación a los resultados de sus alumnos, se logra por las expectativas que este tenga consigo mismo y con sus alumnos, en la UCSH debemos pensar en cómo educar a quienes ingresan a una carrera profesional con expectativas bajas y a veces con desmotivaciones, que afectan los resultados en una carrera. Quizás sea este elemento en el proceso de ingreso, que provoque al mismo tiempo, una reversión en los estudiantes, en la imagen de sí mismos y de la propia universidad.

Por una cuestión de principios identitarios y carismáticos la UCSH comprende un modo de ser entre quienes participan de la comunidad educativa, como con quienes recién ingresan. La amabilidad, el buen trato, la acogida, la alegría y la sana convivencia, son elementos que ayudan y promueven el desarrollo de las motivaciones y expectativas que trae un estudiante. Son elementos que en el quehacer académico les permite desarrollar, por ejemplo, dinámicas de trabajo en equipo, capacidad de resolver comunitariamente y al mismo tiempo crecer en las expectativas personales en el proyecto de vida. Las mismas características que anuncian los estudiantes como indicadores para estudiar pedagogía.

Sin embargo, los elementos nombrados, según indican los discursos, no logran proyectar una imagen de carrera acorde a las expectativas que genera un ambiente de acogida o las relaciones humanas amables y alegres. La carrera docente sigue teniendo una imagen de ser un trabajo complicado, una pega jodida, sea por las evaluaciones docentes, por los malos sueldos o por los bajos resultados en evaluaciones estándares. Hay aquí otro elemento a discernir en la formación de los estudiantes, que ganando espacio y logros en sus motivaciones, puedan trabajar fuertemente en la cultura del desempeño y trabajo de calidad, que perfecciona en el cotidiano lo que debe proyectar con los demás.

Nuevamente la cuestión identitaria y carismática pone en juego la posibilidad de hacer crecer y optimizar el potencial que cada 
estudiante tiene en esta universidad. El reconocimiento como personas que anhelan desarrollar una misión en el futuro, anima a docentes y a los mismos estudiantes a significar permanentemente sus opciones. Estas características del sello identitario permiten a los estudiantes validarse como autónomos en la diversidad discursiva en la que están insertos.

La autonomía de cada estudiante, en la diversidad discursiva, en la pluralidad de opciones y calidad de los argumentos que justifican cada opción de un estudiante, la universidad aparece como una oportunidad de ser un profesional en medio de quienes tienen el mérito de serlo. En los estudiantes de UCSH, se juega un mérito no obtenido o mejor aún, no valorado por los bajos resultados de la PSU, que al mismo tiempo, construyen una imagen social negativa sobre los profesionales de la educación. Se juega en los estudiantes de la UCSH un abrirse camino, allí donde los caminos parecían cerrados. La esperanza, la capacidad de atreverse en un espacio que los acoge y los significa como estudiantes, permite comprender que la opción de ingreso de los alumnos esté garantizada.

\section{Bibliografía}

Basombrío, M. (2008). De la filosofía del yo a la bermenéutica del sí mismo. Servicio de publicaciones de la Universidad de Málaga.

Bernstein, B. (I990). La construcción social del discurso pedagógico. Cap. 3. "Códigos Elaborados y Restringidos: Revisión y Crítica”. Bogotá, El Griot: 8III7.

Boscán, I. (2008). "El saber pedagógico del docente, una reconstrucción biográfica". Teré: revista de filosofía y socio-política de la educación, N. 7, 2008: 99-I06.

Martinic, S. (2005). Presentación Método de Análisis estructural para el estudio de las representaciones sociales. Material de apoyo a la docencia. Documento en revisión. PUC.

Mineduc (2005). Informe Comisión sobre Formación Inicial Docente. Serie Bicentenario.

Moscovici, S. (1986). Psicología Social II. Cap. Denise Jodelet, "La representación social: fenómenos, conceptos y teoría”. Barcelona, Paidós: 47I-493. 
Narración de estudiantes de pedagogía ante el proceso de ingreso a la universidad - Antonio Freire, Nelson Rodríguez y Pablo Solórzano

Restrepo, A. (2004). "La investigación-acción educativa y la construcción de saber pedagógico”. Bernardo Gómez. Educación y educadores No 7: 45-56.

Ricoeur, P. (I987). Tiempo y narración. Vol. I y II. Madrid: Ediciones Cristiandad.

Ricoeur, P. (1977). La metáfora viva. Buenos Aires: Editorial La Aurora.

Ricoeur, P. (1984). Educación y política: de la bistoria personal a la comunicación de libertades. Buenos Aires: Docencia.

Ricoeur, P. (1999). Historia y narratividad. Barcelona: Paidós.

Ricoeur, P. (1995). Teoría de la interpretación: discurso y excedente de sentido. México: Siglo Veintiuno.

Ricoeur, P. (1983). Texto, testimonio y narración. Santiago de Chile: Editorial Andrés Bello.

Solar, M. I. y Díaz, C. (2009). "El profesor universitario: construcción de su saber pedagógico e identidad profesional a partir de sus cogniciones y creencias". Calidad en la educación, $N^{\circ}$ 30: 207-232.

Vieytes, R. (2004). Metodología de la Investigación. Epistemología y técnicas. Buenos Aires: Editorial de Las Ciencias.

Zapata, V. (2003). Revista Educación y Pedagogía Vol. I5, N. 37, 2003 (Ejemplar dedicado a: Foucault, la educación y la pedagogía: a los veinte años de la muerte de Michael Foucault, en homenaje a Alberto Restrepo): I75-I84. 